\title{
Quantum Model for Metal-Insulator Transition in Amorphous $\mathrm{Gd}_{x} \mathrm{Si}_{1-x}$
}

\author{
A. PAJA* AND M. ORNAT
}

AGH University of Science and Technology, Faculty of Physics and Applied Computer Science Department of Solid State Physics, al. A. Mickiewicza 30, 30-059 Krakow, Poland

(Received February 6, 2013; in final form July 4, 2013)

\begin{abstract}
The model for experimentally observed metal-insulator transition induced by magnetic field in amorphous $\mathrm{Gd}_{x} \mathrm{Si}_{1-x}$ is presented. The method of calculation is based on the previously created model for amorphous alloys now developed to include magnetic field effects. The model is based on the quantum " $2 k_{\mathrm{F}}$ " scattering model theory where the pseudopotentials are replaced by the scattering matrix operators and the Fermi energy is properly determined by the accurate values of the phase shifts. The results agree very well with experimental data.
\end{abstract}

DOI: $10.12693 /$ APhysPolA.124.717

PACS: 72.15.Cz, 72.15.Lh, 72.15.Rn

\section{Introduction}

Magnetic field can induce metal-insulator transition in highly disordered systems, that was experimentally found for amorphous $\mathrm{Gd}_{x} \mathrm{Si}_{1-x}$ at the temperatures close to absolute zero by Teizer et al. [1]. Teizer and other researchers investigated this material and its various properties in later papers [2-4] but they did not explain that transition. Hereafter we show how such transition can be explained within the model of the electron transport that we presented in our previous work [5]. To our knowledge it is the first explanation of this effect based on the quantum transport theory of disordered systems.

Previously we developed the model [5] for calculations of the resistivity of disordered systems basing on the Morgan-Howson-S̆aub (MHS̆) model with the Evans correction which improved its stability and precision. We corrected the Esposito method for the Fermi energy determination by improving the phase shifts calculations and finally we obtained two-parameter, fully quantum model, that can be used for multi-element alloys. The performed calculations have shown that the model can be considered as universal one for a quite large group of simpler elements.

In the next section we show how this model can be extended to include magnetoconductivity effects as it was performed for the original MHS model [6]. After this modification we use the model for calculations of the conductivity in $\mathrm{Gd}_{0.14} \mathrm{Si}_{0.86}$ system at $0 \mathrm{~K}$ temperature to present our explanation of the observed metal-insulator transition in this material.

\section{Model}

The resistivity is calculated as in [5]:

$$
\rho=\frac{m_{\mathrm{e}}^{*}}{n e^{2} \tau_{\mathrm{tr}}},
$$

where

*corresponding author; e-mail: paja@agh.edu.pl

$$
\frac{1}{\tau_{\mathrm{tr}}}=\frac{1}{\tau_{\mathrm{FZ}}} \frac{1+\frac{1}{2} \frac{\tau_{\mathrm{FZ}}}{\tau} X^{2} F_{\mathrm{MHS}}^{1 / 2}(X)}{1-\frac{3}{64} X^{4} F_{\mathrm{MHS}}(X)} .
$$

Here $\tau_{\mathrm{FZ}}$ stands for the transport lifetime and $\tau$ is one-electron lifetime (both taken from the Faber-Ziman (F-Z) model $), X=\hbar /\left(\tau E_{\mathrm{F}}\right)$, and

$$
\begin{aligned}
& F_{\mathrm{MHS}}(X)= \\
& {\left[2 \ln \frac{\left(1+X^{2}\right)^{1 / 2}+1+2^{1 / 2}\left[\left(1+X^{2}\right)^{1 / 2}+1\right]^{1 / 2}}{\left(1+X^{2}\right)^{1 / 2}+1-2^{1 / 2}\left[\left(1+X^{2}\right)^{1 / 2}+1\right]^{1 / 2}}\right]^{2} .}
\end{aligned}
$$

Instead of the original formula for $\mathrm{F}-\mathrm{Z}$ lifetimes we follow Evans et al. [7] taking

$$
\begin{aligned}
& \frac{1}{\tau_{\mathrm{FZ}}}=\frac{m_{\mathrm{e}}^{*} \Omega_{0}}{4 \pi \hbar^{3} k_{\mathrm{F}}^{3}} \int_{0}^{2 k_{\mathrm{F}}} \mathrm{d} q \lambda(q) q^{3}, \\
& \frac{1}{\tau}=\frac{m_{\mathrm{e}}^{*} \Omega_{0}}{2 \pi \hbar^{3} k_{\mathrm{F}}} \int_{0}^{2 k_{\mathrm{F}}} \mathrm{d} q \lambda(q) q,
\end{aligned}
$$

where $\Omega_{0}$ is the average volume per one atom in the system, and for $p$-element alloy we generalized $\lambda(q)$ to be equal to

$$
\lambda(q)=\sum_{\alpha=1}^{p} \sum_{\beta=1}^{p} \sqrt{c_{\alpha} c_{\beta}} S_{\alpha \beta}(q) t_{\alpha}(q) t_{\beta}^{*}(q) .
$$

In this equation $c_{i}$ stands for the number concentration of the $i$-th component, $S_{i j}$ are the Ashcroft-Langreth partial structure factors [8] and $t_{i}(q)$ are scattering operator matrix elements (at the Fermi level):

$$
t(q)=-\frac{2 \pi \hbar^{3}}{\Omega_{0} m_{\mathrm{e}}^{*} k_{\mathrm{F}}} \sum_{l}(2 l+1) \sin \eta_{l} \mathrm{e}^{\mathrm{i} \eta_{l}} P_{l}(\cos \theta) .
$$

where $P_{l}$ are the Legendre polynomials, $\cos \theta=1-$ $q^{2} /\left(2 k_{\mathrm{F}}\right)^{2}$, and $\eta_{l}-$ phase shifts at the Fermi level for the $l$-th band of the considered element.

\subsection{Structure factors and phase shifts}

Details of the methodology used for the calculation were presented in [5]. We use the Ashcroft-Langreth partial structure factors [8] calculated by means of the method presented by Hoshino [9] and also we follow 
Waseda empirical rule [10] for calculation of the packing fraction. Phase shifts are calculated by solving the Schrödinger equation with the muffin-tin potential calculated by means of the Mattheiss prescription generalized for disordered systems by Mukhopadhyay et al. [11, 12] and with counting wave function oscillations to obtain their proper values as it was described in [5].

\subsection{Magnetic field effects}

Magnetic field influence on the resistivity is calculated as in generalized " $2 k_{\mathrm{F}}$ " model [6] that leads to

$$
\tau_{\mathrm{T}}^{-1}=\tau^{-1}+\eta^{-1}(\boldsymbol{B}),
$$

where $\tau_{\mathrm{T}}$ stands for the total relaxation time and

$$
\eta^{-1}(\boldsymbol{B}) \approx-\lambda B^{2}
$$

where $\lambda \approx 10^{11} \mathrm{~T}^{-2} \mathrm{~s}^{-1}$ is a parameter of the model.

It was shown that transport lifetime in the generalized model can be calculated as [6]:

$$
\frac{1}{\tau_{\mathrm{tr}}^{*}}=\frac{1}{\tau_{\mathrm{tr}}^{\mathrm{FZ}}} \frac{1+\frac{1}{2} \frac{\tau_{\mathrm{tr}}^{\mathrm{FZ}}}{\tau} \frac{Y^{3}}{X} \sqrt{F_{\mathrm{MHP}}(Y)}}{1-\frac{3}{64} X Y^{3} \sqrt{F_{\mathrm{MHS}}(X)} \sqrt{F_{\mathrm{MHP}}(Y)}},
$$

where $X=\hbar /\left(E_{\mathrm{F}} \tau\right)$ and $Y=\hbar /\left(E_{\mathrm{F}} \tau_{\mathrm{T}}\right)=X\left(1+\tau \eta^{-1}\right)$.

Function $F_{\mathrm{MHP}}^{1 / 2}(Y)$ for $A>0$ is expressed as

$$
F_{\mathrm{MHP}}^{1 / 2}(Y)=
$$

$\ln \left(\frac{y+1+\sqrt{2(y+1)}+2 Y \sqrt{\frac{A}{1-A}} \sqrt{y-1}+2 Y^{2} \frac{A}{1-A}}{y+1-\sqrt{2(y+1)}+2 Y \sqrt{\frac{A}{1-A}} \sqrt{y-1}+2 Y^{2} \frac{A}{1-A}}\right)^{2}$

and for $A<0$ :

$$
F_{\text {MHP }}^{1 / 2}(Y)=
$$

$\ln \frac{\left[y+1+2 Y^{2} \frac{|A|}{1-A}+\sqrt{2(y+1)}\right]^{2}-2 Y^{2} \frac{|A|}{1-A}[\sqrt{2(y+1)}+2]^{2}}{\left[y+1+2 Y^{2} \frac{|A|}{1-A}-\sqrt{2(y+1)}\right]^{2}-2 Y^{2} \frac{|A|}{1-A}[-\sqrt{2(y+1)}+2]^{2}}$, where $A=1-\frac{\tau_{\mathrm{T}}}{\tau}$ and $y=\sqrt{1+Y^{2}}$.

This definition allows for calculation of the magnetoconductivity as

$$
\Delta \sigma_{\mathrm{M}}(B, T)=\sigma(B, T)-\sigma(0, T) .
$$

Finally, to obtain resistivity in presence of the magnetic field we add the result above to the regular conductivity

$$
\frac{1}{\rho(B)}=\frac{1}{\rho_{\mathrm{MHS}}}+\frac{1}{\rho_{\mathrm{MHP}}(B)}-\frac{1}{\rho_{\mathrm{MHP}}(B=0)},
$$

where the conductivities are replaced by the inverse resistivities, $\rho$ is the final resistivity, $\rho_{\text {MHS }}$ is the resistivity within the regular model without magnetic field effects included as presented in the beginning of the section and $\rho_{\mathrm{MHP}}$ is the resistivity within the model generalized for magnetic effects as presented above.

\section{Results}

We performed calculations within the presented model for $\mathrm{Gd}_{0.14} \mathrm{Si}_{0.86}$ amorphous system at $0 \mathrm{~K}$ temperature. Parameters used for calculations were taken as: $\lambda=$
$1.23 \times 10^{12} \mathrm{~T}^{-2} \mathrm{~s}^{-1}, \alpha_{\mathrm{Gd}}=0.7, \alpha_{\mathrm{Si}}=0.9045$, and the effective to regular electron mass ratios were assumed to be equal to one.

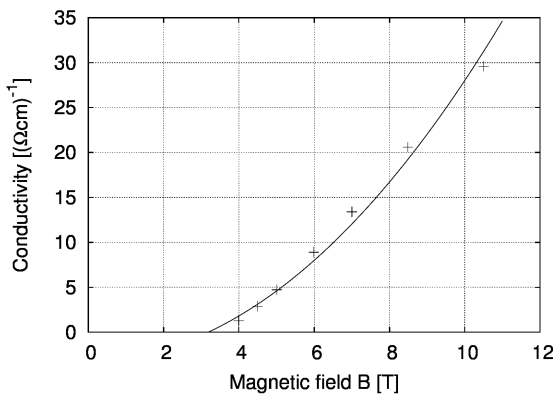

Fig. 1. Magnetic field induced metal-insulator transition in $\mathrm{Gd}_{0.14} \mathrm{Si}_{0.86}$ system at $0 \mathrm{~K}$ temperature. Solid line denotes our calculation results and crosses are the experimental data taken from [1].

Results are presented in Fig. 1. We can see very good agreement between our calculations and the experimental data, which suggests that the physics of the model is correct and the model can be used for such calculations. These results show that the observed phenomenon can be explained as the effect of destruction of the quantum interference that occurs as a result of high disorder in the system, and so it can be seen as the Anderson transition.

\section{Discussion}

Calculations were performed with the use of the simplest possible set of parameters. In fact, neither effective electron masses nor the Slater exchange rate $\alpha_{\mathrm{Gd}}$ were adjusted in any way and their values were taken as the most typical ones. Only two parameters were used for fitting: the Slater exchange rate $\alpha_{\mathrm{Si}}$, the value of which was established reasonably in the range 0.6 to 1.0 , and the parameter $\lambda$ adopted from the MHS model generalized in [6]. It describes the effect of the magnetic field on the conductivity and it also turned out to have sound value.

Anyway the fitting was performed for the single case of the $\mathrm{Gd}_{0.14} \mathrm{Si}_{0.86}$ amorphous alloy and was not verified for any other system. This should not affect our conclusions but only means that the values of parameters may be different in calculations for other systems.

\section{Summary}

We have adjusted previously developed and verified model to include the influence of magnetic field on the transport properties of disordered systems. We performed calculations of the magnetoconductivity for amorphous $\mathrm{Gd}_{0.14} \mathrm{Si}_{0.86}$ system for which the metal-insulator transition in the external magnetic field was experimentally observed. We have obtained very good agreement between our results and the experimental data, which 
suggests that the model can successfully be used for such systems. Our result shows that the considered transition can be explained as the effect of destruction of the quantum interference by magnetic field and therefore can be regarded as a kind of the Anderson transition.

\section{Acknowledgments}

The work was partially supported by the Polish Ministry of Science and Higher Education and its grants for Scientific Research (grant number 11.11.220.01).

\section{References}

[1] W. Teizer, F. Hellman, R.C. Dynes, Solid State Commun. 114, 81 (2000).

[2] W. Teizer, F. Hellman, R.C. Dynes, Physica E 18, 266 (2003).

[3] L. Bokacheva, W. Teizer, F. Hellman, R.C. Dynes, Phys. Rev. B 69, 235111 (2004).
[4] S. Caprara, V.V. Tugushev, Microelectron. Eng. 81, 293 (2005).

[5] M. Ornat, A. Paja, Appl. Phys. A: Mater. Sci. Proc. 102, 379 (2011).

[6] A. Paja, G.J. Morgan, Phys. Status Solidi B 208, 701 (1998).

[7] R. Evans, D.A. Greenwood, P. Lloyd, Phys. Lett. 35A, 57 (1971).

[8] N.W. Ashcroft, D.C. Langreth, Phys. Rev. 159, 685 (1967).

[9] K. Hoshino, J. Phys. F, Met. Phys. 13, 1981 (1983).

[10] Y. Waseda, The Structure of Non-Crystalline Materials, McGraw-Hill, New York 1980.

[11] L.F. Mattheiss, Phys. Rev. 133, A1399 (1964).

[12] G. Mukhopadhyay, A. Jain, V.K. Ratti, Solid State Commun. 13, 1623 (1973). 